\title{
Vivir la frontera. Prácticas sociales y culturales desde los márgenes
}

\section{Living the border. Social and cultural practices from the sidelines}

Mariana Winikor Wagner ${ }^{*}$ (ID (http://orcid.org/0000-0001-8166-0112)
Recibido el 23 de septiembre de 2015.

Aceptado el 28 de abril de 2016.

*Autor para correspondencia: Mariana Winikor Wagner, correo electrónico: marianawinikorwagner@gmail.com

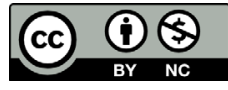

Todos los contenidos de Estudios Fronterizos se publican bajo la licencia Creative Commons Atribución no comercial 2.5 México, y pueden ser usados gratuitamente para fines no comerciales, dando el crédito a los autores y a la revista Estudios Fronterizos.
${ }^{1}$ Universidad Nacional de Misiones, Universidad de Buenos Aires, Consejo Nacional de Investigaciones Científicas y Técnicas, Buenos Aires, Argentina, correo electrónico: marianawinikorwagner@gmail.com

\section{Resumen}

Los migrantes, en tanto ocupan una posición de subalternidad y vulnerabilidad en la sociedad de destino, pueden ser definidos como sujetos de análisis de los estudios culturales. Es desde este marco teórico, que indagaremos las prácticas sociales y culturales de los migrantes brasileños asentados en el nordeste de la provincia de Misiones, Argentina. Enfocaremos nuestra atención en analizar si estas prácticas son verdaderamente formas de resistir a la cultura dominante o simplemente constituyen formas de reproducción y naturalización de las mismas. A través de cuatro prácticas concretas (el portuñol, el brique, la compra-venta de mejoras y la ocupación espontánea de tierras privadas) llegaremos a la conclusión de que las mismas evidencian préstamos, concesiones, amalgamas, conflictos, subordinación y a la vez intersticios de creatividad con la cultura dominante. Al mismo tiempo, demuestra el carácter relacional que posee la cultura popular con respecto a la cultura hegemónica y su posición de subalternidad y de dominación.

Palabras clave: frontera, prácticas, migrantes, cultura popular, hegemonía.

\section{Abstract}

Migrants, while in a position of subordination and vulnerability in the host society, can be defined as subjects of analysis of cultural studies. It is from this theoretical framework, which will investigate the social and cultural practices of Brazilian migrants settled in the northeast of the province of Misiones, Argentina. We focus our attention on analyzing whether these practices are truly ways to resist the dominant culture or simply constitute forms of reproduction and naturalization of them. Through four concrete practices (portuñol, el brique, the buying and selling of improvements and

CÓMO CITAR: Winikor, M. (2016). Vivir la frontera. Prácticas sociales y culturales desde los márgenes [Living the border. Social and cultural practices from the sidelines]. Estudios Fronterizos, 17(34), 100-116, https://doi.org/10.21670/ref.2016.34.a06 
spontaneous occupation of private land) will reach the conclusion that the same evidence loans, grants, amalgams, conflicts, subordination and yet interstices creativity with the dominant culture. It shows the relational character that owns the popular culture with the dominant culture and its position of subordination and domination.

Keywords: border, practices, migrants, hegemony, popular culture.

\section{Introducción}

En este trabajo, nos enfocaremos en el análisis de ciertas prácticas culturales de los migrantes brasileños asentados en El Soberbio, un pueblo de frontera al nordeste de la provincia de Misiones (Argentina). Definidos por su condición de subalternidad en tanto ocupan una posición de dominación en la sociedad receptora, los migrantes componen las denominadas clases populares permitiendo encuadrar este estudio en el marco de los estudios culturales.

Describiremos algunas prácticas típicas de los pequeños productores y las analizaremos en relación a su posición frente a la cultura hegemónica. ¿Son estas prácticas formas de resistir a la cultura dominante o simplemente constituyen formas de reproducción y naturalización? ¿Pueden ser interpretadas como un momento de producción simbólica autónoma? Estas preguntas concentran algunas de las líneas analizadas en este artículo.

En el primer apartado se presenta la introducción, enseguida, explicitaremos la metodología utilizada; luego abordaremos los debates académicos existentes en los estudios culturales desarrollados en nuestro país, que nos permitirán comprender a qué nos referimos con cultura popular y qué se entiende por estudios culturales. En la cuarta parte del trabajo justificaremos por qué los migrantes pueden ser sujetos de análisis de los estudios sobre cultura popular. En el quinto apartado caracterizaremos brevemente la localidad analizada, para luego desarrollar el análisis de cuatro prácticas culturales características de los migrantes brasileños asentados en la zona de frontera: la utilización del portuñol, el brique, la compra-venta de mejoras y la ocupación espontánea de tierras privadas; culminando con la aportación de nuestras reflexiones finales.

\section{Metodología}

Los datos y resultados aquí expuestos fueron resultado de un proceso de investigación desarrollado mediante la aplicación de técnicas y métodos cualitativos de carácter socioantropológico. Combinamos el análisis de fuentes primarias recolectadas a través de la entrevista en profundidad, la observación participante y notas de campo, con el análisis de fuentes cualitativas secundarias provenientes de autores que anteriormente han indagado la zona de frontera analizada.

El trabajo de campo se llevó a cabo durante el año 2015 en Colonia Monteagudo y el casco periurbano de El Soberbio, ambos sitios donde residen pequeños productores de origen brasileño. La elección de Colonia Monteagudo radica en que es una colonia histórica de la localidad por su incipiente poblamiento llevado adelante por migrantes de origen brasileño. Por este motivo, Monteagudo fue el primer lugar del municipio con presencia del Estado nacional lo que lo lleva a asumir la cabecera del Departamento Guaraní. En 1946, y como consecuencia de la llegada de pequeños productores a la zona urbana y periurbana del pueblo, es El Soberbio quien asume ese lugar. 
Se realizaron 11 entrevistas en profundidad orientadas a productores, docentes, migrantes y sorberbianos ${ }^{2}$ en general y, tres instancias de observación participante en familias de pequeños productores, en las cuales pudimos analizar las prácticas sociales y culturales que se desarrollarán en este documento.

\section{Debates en torno a la cultura popular}

Indagar sobre la cultura popular implica necesariamente definir ciertos conceptos que en el campo académico de los estudios culturales se encuentran aún en debate. Reflexionar sobre qué es lo popular y a qué nos referimos con cultura popular resulta entonces indispensable.

Estudiar lo popular es preguntarse por las relaciones de poder y dominación, la exclusión y la desigualdad social; es indagar las prácticas de las que hacen uso los sectores populares para hacer frente a esa situación de subalternidad que pueden derivar en prácticas de resistencia — que se propongan hacer evidente la relación de dominación o modificarla-, naturalización o reproducción de la dominación, según el caso; es indagar sobre la dimensión simbólica de las prácticas de los sectores populares (Rodríguez, 2011). Lo popular debe entenderse en términos gramscianos desde un punto de vista relacional que se pregunta por el vínculo de las clases populares con el resto de los sectores sociales en pugna por la hegemonía (Aliano, 2010).

En Argentina, los estudios sobre cultura popular definen "lo popular entendido como subalterno" (Alabarces y Añón, 2008, pp. 282), o como dirían Albarces, Añón y Conde (2008, p. 8) "subalternidad conflictiva y desplazada"; surgen de una necesidad política de la época de instalar lo popular como tópico, de rescatar objetos de estudio periféricos, no centrales dentro del campo legítimo de la cultura. Ford, Romano y Rivera, abren el camino de lo que posteriormente la academia denominara estudios culturales, preguntándose por aquello que está fuera de lo visible y de lo enunciable, en síntesis, condenados al olvido (Alabarces et al., 2008).

Definimos cultura popular como el conjunto de experiencias, prácticas, representaciones de los sectores populares que se encuentran en constante disputa por el sentido, que resultan específicas de su modo de percibir y vivir la realidad. Es conveniente referirnos a las culturas populares en plural para dar cuenta de la heterogeneidad de experiencias pertenecientes a los sectores populares, contradiciendo la mirada legítima que le otorga a todas ellas rasgos homogéneos (Rodríguez, 2011). ${ }^{3}$

Una vez instalada la temática, surgen diversas líneas de abordaje al interior del campo teórico de las culturas populares en nuestro país. Por un lado, perspectivas que tienen como objetivo analizar de forma relacional la condición de subalternidad, las formas de resistencia o de reproducción de las clases subalternas, que se orientan a demostrar los efectos de la dominación; y por el otro, enfoques que ponen el acento en la positividad de las culturas populares, que muestran la capacidad de estos sectores de producir un sistema simbólico coherente y autónomo sin negar los efectos de la dominación simbólica (Aliano, 2010).

\footnotetext{
${ }^{2}$ Gentilicio que refiere a los habitantes que nacieron en El Soberbio.

${ }^{3}$ Resulta imprescindible aclarar que los estudios culturales no son exclusividad de las clases populares. Sectores medios y medios-altos también son sujetos de análisis dado el frecuente proceso de plebeyización de la cultura. Por lo tanto, no es una cuestión de clase sino de posición frente a la cultura dominante.
} 
Nos situamos en una posición intermedia, pues si bien creemos en la capacidad de agencia de los sectores populares y en sus intersticios de "autonomía" creativa, consideramos que el análisis de esas prácticas, valores y representaciones culturales deben siempre pensarse en términos relacionales, reconociendo la lucha simbólica permanente de las clases populares por la hegemonía (Alabarces y Añón, 2008). Como afirma Hall (como se citó en Aliano, 2010, p. 189) 'lo esencial para la definición de la cultura popular son las relaciones que definen a la 'cultura popular' en tensión continua (relación, influencia y antagonismo) con la cultura dominante". ${ }^{4}$ Creemos que la cultura en general (y la cultura popular en particular) no tiene existencia autónoma, dado que la trama cultural se conforma de negociaciones, apropiaciones y rechazos múltiples con la cultura oficial. El estudio de las culturas populares está marcado por su condición de subalternidad y asimetría al interior del campo social, por lo que resulta indispensable analizar si estas prácticas implican ciertas operaciones de resistencia en la lucha simbólica o simplemente pretenden reproducir determinadas prácticas oficiales. Siempre analizaremos el accionar de las clases populares en clave política, en relación a su posición de dominación que implica conflicto y lucha por el sentido.

La tendencia académica de asimilar, en las sociedades altamente mediatizadas, cultura popular a cultura masiva, y considerar que la experiencia popular no puede concebirse por fuera de esta matriz, imposibilita el abordaje de determinadas prácticas subalternas locales, rurales y de los márgenes que se encuentran por fuera del mercado y la industria cultural. No toda cultura está mediada por la industria cultural. Creemos que esta perspectiva se orienta a analizar experiencias estrictamente urbanas $^{5}$ (podríamos calificarla de urbanocentristas) obstaculizando el tratamiento de aquellas prácticas que quedan por fuera de la influencia masiva de los medios de comunicación. Como afirma Aliano (2010) la heterogeneidad de lo que entendemos como cultura popular no puede disolverse en lo masivo. La cultura popular rural adquiría la categoría de "lo subalterno de lo subalterno", quedando directamente fuera del análisis de los estudios culturales. Las prácticas y experiencias que abordaremos en este trabajo no son masivas sino locales (y fronterizas), sin embargo, pertenecen a lo que Gramsci (2004) denomina pueblo, en cuanto conjunto de clases subalternas. Reconocemos las fisuras que se encuentran al interior de lo que definimos como clases populares dado que de ninguna manera podemos concebirlas como un todo homogéneo; por ello proponemos ampliar el foco de atención y abordar ciertos objetos excluidos por varios académicos de la materia. Decidimos entonces, analizar ciertas prácticas sociales y culturales de los migrantes brasileños asentados en colonias rurales $^{6}$ de la frontera nordeste de Misiones con el objetivo de enunciar y dar espacio en el ámbito de los estudios culturales a estos actores sociales, sus experiencias y prácticas de frontera.

\footnotetext{
${ }^{4}$ Para este autor, la cultura popular está enmarcada en determinadas relaciones de producción, permeable a las disputas por el sentido.

${ }^{5} \mathrm{Si}$ bien afirmamos el tratamiento del folklore y la gauchezca dentro de los estudios culturales en nuestro país, su abordaje se debió a los intereses políticos existentes dentro de la clase dominante, lo que dio espacio a estos tópicos - la necesidad de conformar una identidad nacional, que homogeneíce a la masa inmigratoria de ultramar y se imponga como dominante frente a la "barbarie" del interior-. Sin embargo, el análisis de la cultura rural en tanto cultura popular estuvo desatendida y permanentemente fuera de foco.

${ }^{6}$ Se denomina colonias a los asentamientos rurales fruto del poblamiento llevado a cabo por programas de colonización tanto oficiales, privados como espontáneos.
} 


\section{La migración como subalternidad}

Lo popular implica la existencia de múltiples formas jerárquicas que admiten la situación de subalternidad, que dependerán de las especificidades históricas del caso analizado: casta, género, clase, ocupación, etnia, o cualquier otra forma posible de dominación (Guha, citado en Alabarces y Añón, 2008), que puede dar origen a una relación asimétrica. Entonces nos preguntamos si la migración puede dar origen a una relación de este tipo.

El proceso migratorio coloca a los sujetos migrantes en una situación de vulnerabilidad en la sociedad de destino por varias circunstancias. Por un lado, por el solo hecho de encontrarse lejos de su comunidad y cultura de origen, en un contexto ajeno y extraño; asimismo, por la situación jurídica migratoria precaria en la que se encuentran muchos migrantes (que implica integrarse al mercado de trabajo informal caracterizado por bajos salarios, malas condiciones de empleo — muchas veces determinada por la explotación laboral y condiciones que se asemejan a la esclavitud-) $;^{7}$ por las estrategias familiares puestas en juego al momento de migrar (que dependiendo de la modalidad "elegida" implica la separación del migrante de su núcleo familiar primario); y por último, dependiendo del país receptor, de los derechos otorgados a la población migrante. Por el otro, por la existencia de una sociedad nativa que muchas veces refuerza esas relaciones de poder, poniendo en funcionamiento una maquinaria basada en prácticas discriminatorias y disciplinamientos sociales hacia determinadas colectividades de migrantes. Estas manifestaciones son ejercidas como forma de mantener vigente e intacta la identidad nacional y la estructura social en aquellas circunstancias que pueden amenazarla, permitiendo a determinados grupos dominantes (nativos en este caso) perpetuarse ideológica y socialmente (Winikor, 2013). Como afirma Cohen (2009) se adopta un discurso de tolerancia hacia la diversidad cultural, a partir de relaciones de poder y dominación que si bien reconocen al migrante, le establecen límites y obstáculos para su integración. Un espacio social basado en relaciones sociales asimétricas de desigualdad y exclusión, que le otorga al migrante el lugar de extraño en vez de par, acrecentándose esa frontera entre migrantes y nativos.

La sanción en nuestro país de la Ley de Migraciones No. 25.781 (2004), en el año 2003, tiene como objetivo explicitar los derechos de los migrantes que residen en Argentina (haciendo hincapié en los derechos relacionados al acceso a la salud, educación, reunificación familiar, etc.) y promover la integración social de los mismos, igualando los derechos de los migrantes al de los nacionales. La necesidad de crear una ley de este tipo evidencia la desigualdad de trato existente hacia los extranjeros en la sociedad argentina, haciendo visible la situación de vulnerabilidad que presentan los mismos en su calidad de migrantes. Ninguna ley surge si no es necesario reivindicar ciertos derechos y obligaciones.

Definimos entonces a los migrantes dentro de lo que Gramsci (2004), denomina el conjunto de las clases subalternas por esta doble condición de vulnerabilidad y subalternidad ${ }^{8}$

\footnotetext{
${ }^{7}$ Prácticas llevadas adelante no solo por la sociedad receptora sino por miembros de su propia comunidad que se encuentra asentados con anterioridad en la sociedad de destino y desarrollan los denominados enclaves étnicos.

${ }^{8}$ Resulta necesario explicitar que no todos los migrantes ocupan una posición subalterna en la sociedad de destino como tampoco son homogéneas las representaciones sociales de los nativos en relación al conjunto de inmigrantes. A través del análisis discursivo de los nativos (Winikor, 2013) pudimos identificar la existencia de migrantes "deseables" relacionados a los provenientes de naciones ricas y migrantes "indeseables" relacionados a los países pobres, lo que en el marco de los estudios migratorios se conoce como jerarquización de naciones (Pottilli, Silberstein y Tavernelli, 2009).
} 
(la intrínseca correspondiente al propio proceso migratorio y la impuesta por la sociedad receptora) a la que se le suma una tercera íntimamente relacionada a estas dos: las condiciones socioeconómicas en las que se encuentran varios colectivos migratorios en el país de destino (que en muchos casos es peor a las condiciones que enfrentaban en sus países de origen) y que refuerza las relaciones de poder y las representaciones discriminatorias puestas en funcionamiento por la sociedad nativa. Se invisibiliza el rechazo de los nativos hacia las clases más desposeídas a través de discursos racializados, lo que se conoce como racialización de las condiciones de clase. Frecuentemente lo que molesta es que los migrantes sean pobres, poniéndose en funcionamiento una administración diferencial de la tolerancia dependiendo del origen nacional de los migrantes.

Por todo lo expuesto anteriormente, las características que asumen los migrantes permitirían definir sus prácticas culturales en términos de cultura popular.

\section{Vivir en los márgenes}

El Soberbio es una localidad fronteriza ubicada al nordeste de la Provincia de Misiones, que limita con la República Federativa del Brasil a través del río Uruguay. Si bien cuenta con Paso Internacional Fronterizo oficial que comunica con su pueblo gemelo Porto Soberbo, la mayoría de los soberbiarnos frecuentan el paso por motivos económicos, familiares, laborales y comerciales a través de los porto capiovaras, ${ }^{9}$ como se conoce a las formas de transitar la frontera e ingresar al país a través de puertos ilegales a lo largo del río. La frontera entonces, no podemos definirla como un límite político-administrativo, como una línea, sino como una región de movilidad continua, que permite que emerja una cultura de frontera tendiente a invisibilizar la división político territorial y a exhibir el desajuste entre los límites del Estado y de la nación. El portuñol utilizado por la comunidad que habita a un lado y al otro del río, que impregnan la modalidad de trabajar la tierra y de denominar las técnicas de trabajo; la música que suena en las radios con su impronta brasileña; las comidas típicas que no nos permiten identificar de qué lado del río nos encontramos; la existencia de familias desparramadas en ambos márgenes —entre otras cosas-, evidencia una cultura compartida que excede los límites estatales.

Según el Censo del año 2010, El Soberbio cuenta con una población total de 22898 habitantes, donde $77.9 \%$ es de origen rural (Instituto Nacional de Estadística y Censos [Indec], 2012). Los carros tirados por bueyes configuran el paisaje cotidiano de la localidad y nos invitan a reflexionar sobre esta predominancia de población rural. Son utilizados actualmente por muchos colonos como único medio de transporte que permite acercar las chacras ${ }^{10}$ al pueblo, para proveerse de los bienes necesarios para la vida, y al mismo tiempo nos dice mucho sobre la dificultad de comunicación que presentan las colonias y parajes del municipio.

La población de El Soberbio es en su mayoría inmigrante y descendiente de inmigrantes de origen brasileño (muchos de ellos brasileños de origen alemán conocidos como teutobrasileños) provenientes de las colonias rurales del sur de Brasil, y por misioneros provenientes de colonias históricas del sur y del centro de la provincia. En la localidad también se encuentran varias comunidades mbya guaraní, mayormente asentadas en las cercanías de la Ruta Provincial No 15.

${ }^{9}$ La expresión local refiere a: puerto de carpinchos (Schiavoni, 1998).

${ }^{10}$ Es la denominación que adopta en Misiones la Explotación Agrícola Familiar que incluye el espacio doméstico, el espacio de cultivo y áreas naturales. 
La actividad económica en El Soberbio, se inició a mediados de siglo XIX con la instalación de obrajes madereros en zonas cercanas al río Uruguay, propiedad de grandes latifundistas. En la actualidad, las actividades económicas principales se orientan a la producción agropecuaria: principalmente cultivos industriales como el tabaco (en menor medida la yerba mate y el té), la producción de forrajeras, citronela, cítricos, maíz y tung.

Entre las décadas de 1960-1990 llegaron al municipio un sinnúmero de inmigrantes provenientes de las colonias de los estados del sur de Brasil (especialmente Río Grande do Sul y Santa Catarina, limítrofes con Misiones). Consecuencia del avance del latifundio, del agronegocio y de la ciencia aplicada a la agricultura y debido a la saturación demográfica de las colonias rurales del sur de dicho país, muchos agricultores familiares se vieron obligados a migrar como estrategia de reproducción de su unidad doméstica ante la pérdida de sus tierras. El poblamiento de El Soberbio se realizó mediante la ocupación espontánea de tierras en propiedad privada abandonadas por sus dueños ${ }^{11}$ (Reboratti, 1979; Schiavoni, 1994), sin la intervención planificada por parte del Estado; circunstancia que explica el número tan elevado de ocupantes carentes de títulos de propiedad. Estos residentes, en su mayoría inmigrantes brasileños, son pequeños productores sin tierra, que se asentaron en la provincia y organizaron el espacio territorial en colonias. A pesar de la dificultad para definirlos, el término colonos sigue siendo el más apropiado para la conceptualización de estos actores sociales (Bartolomé, 1982). ${ }^{12}$

\section{Prácticas fronterizas}

\section{El portuñol}

Como afirma Camblong (2005), a lo largo de la frontera con Brasil se registra la vigencia hegemónica del portugués-brasileño con entrecruzamientos con el español, resultado típico de lenguas en contacto. El portuñol como se le conoce, es una especie de lengua híbrida, de mezcla, conformada por el portugués y el español, utilizada por los colonos brasileños que habitan la frontera analizada. Es, entre la población rural, la única lengua conocida por los niños hasta su escolarización, y en el caso de los adultos sin escolarización la única conocida. La frontera es un espacio de procesos interculturales complejos y de mestizajes y ensambles de lenguas, costumbres y prácticas.

Pero la realidad le gana a la ficción y es mucho más compleja de lo que parece. En la práctica, los niños están atravesados por distintas lenguas de acuerdo a sus espacios de sociabilización: varios en sus casas hablan el portuñol o alguna variación del alemán, ${ }^{13} \mathrm{con}$

\footnotetext{
${ }^{11}$ Empresas orientadas a la extracción de madera nativa. A medida que desforestaban una zona específica abandonaban las tierras, hecho que promovió la ocupación de tierras privadas.

${ }_{12}$ Colono, refiere al tipo social agrario relacionado a la expansión del capitalismo rural en zonas hasta el momento marginadas, constituyendo el tipo específico de productor agrario de la provincia. Si bien decidimos incluir a los pequeños productores de la zona de frontera dentro de la tipología propuesta por Bartolomé (1975), los colonos analizados en este trabajo se caracterizan más por llevar adelante un sistema de reproducción que de producción ampliada con acceso a capitalización, los que los asemeja más a la categoría de campesino que a la de colono, por la utilización de mano de obra estrictamente familiar y la producción orientada al autoconsumo (a pesar de dedicar la mayor parte de su tiempo al cultivo de tabaco). Sin embargo, decidimos definirlos como colonos, pues este es el modo en que ellos se reconocen, preponderando las categorías nativas más allá de las categorías teóricas. Los tipos puros no existen, son simplemente tipos ideales con sus impurezas e imperfecciones para definirlos.

${ }^{13}$ Muchos brasileños que llegaron a la zona son de origen alemán que migraron primero a Brasil, y en una segunda instancia hacia Argentina.
} 
los vecinos dialogan en portuñol y en las escuelas aprenden el español, con el agregado del inglés como lengua extranjera, lo que conlleva a que los niños no hablen adecuadamente ni el portugués ni el español (y mucho menos el inglés).

En muchas escuelas de la zona se les prohíbe a los niños hablar entre ellos en portuñol, aunque en los momentos de recreación (recreos, educación física, peleas) aflora como lengua materna que aunque quiera evitarse, estructura indefectiblemente las relaciones y vínculos sociales cotidianos. "Cuando era chica Fernando [el maestro] nos gritaba porque hablábamos en portugués ${ }^{14}[s i c]$, pero él también habla asî" (Wolski, 2015). Esto evidencia una pugna de las lenguas por la hegemonía: si bien todos hablan portuñol (no solo la población rural sino también la urbana) el español se impone como lengua "oficial, legítima y auténtica". Deviene en lengua dominada que desde la escuela se intenta silenciar y reprimir aunque resulten en vano los intentos. "Los prejuicios y descalificaciones hacia este híbrido lingüístico, provienen de las abstracciones gramaticales de lenguas oficiales normalizadas" (Camblong, 2014, p. 9). Se institucionaliza un conjunto de reglas gramaticales consagradas con la finalidad de descalificar a quienes no las conozcan o dominen, son los maestros y profesores los encargados de imponer un aparato represivo sobre qué y cómo se dice, y qué no (Romano, 1973). Camblong considera que los colonos no hablan ni bien ni mal, sino que ellos "hablan portuñol" y proponen políticas educativas que se orienten al respeto por la cultura fronteriza, que tenga en cuenta la idiosincrasia de las poblaciones rurales de frontera y que promueva una educación intercultural bilingüe. Pero, ¿cómo hacerlo sin intentar normalizar el lenguaje? ¿Sin imponer esquemas horarios fijos donde con un toque de timbre se determine cuándo se habla en portugués y cuándo en español? Imponer el uso de la lengua dominante intentando borrar las expresiones "vulgares" es un proceso conocido en nuestro país que modificó la estética del tango avanzado ya el siglo XX y limitó a locutores del interior a trabajar en los medios de comunicación, entre muchas otras consecuencias a la vista. Cualquier política de este tipo es difícil no calificarla de conservadora y totalitaria.

El lenguaje no es un mero medio de reproducción para expresar las ideas, sino que es conformador de las ideas, organiza simbólicamente la vida de los individuos (Cicourel, 1982) - hay huellas del portuñol en las herramientas de trabajo, en la modalidad de trabajar la tierra, en la forma de relacionarse entre los miembros de la familia, en la modalidad de cocinar, etcétera- El portuñol, en tanto estructura la vida cotidiana de las familias rurales podría interpretarse como un intersticio autónomo por parte de estos sectores populares donde perpetúan sus prácticas culturales más allá de los tabúes y condicionamientos sociales. El uso del portuñol pondría de manifiesto, en cierto sentido, la capacidad de agencia de los sectores populares de imponer sus propias reglas de sociabilidad. Como afirmaría Romano (1973), la actitud respecto al lenguaje permite esclarecer la colaboración, complicidad o ruptura de ciertos grupos con los aparatos del sistema.

Pero cabe preguntarse si las dificultades para comunicarse en español —lo que supone pensar la dificultad futura de insertarse laboralmente en otras tareas que no sean la agricultura familiar, por ejemplo- y el tener como única lengua de dominio el portuñol no refuerza la dominación impuesta sobre estas clases más que ser una muestra de su capacidad de agenciamiento. ¿Es posible que una situación que refuerza la dominación sea a la vez una sumisión liberadora, al permitir la permanencia de las costumbres rurales y fronterizas locales? He aquí una famosa paradoja que enmaraña aún más la intervención

\footnotetext{
${ }^{14}$ Refiriéndose al portuñol.
} 
estatal en este lugar del mundo tan particular. La paradoja del dominado (Bourdieu, 2000), en este espacio, se entrelaza con otra paradoja: la que no puede resolver la tensión entre universalismo y particularismo cultural. Paradojas que como sabemos no tienen solución, solo obligan a la reflexión.

Pero más que capacidad de agenciamiento estas prácticas corresponderían a las condiciones histórico-concretas de habitar la frontera. En palabras de Camblong:

Una espacialidad de tránsitos en las que entran en interacciones lenguas oficiales (español, guaraní y portugués), monedas, símbolos patrios, documentos de identidad, ciudadanías dobles o triples, tensiones y rivalidades históricas, deportivas y políticas, afectos y broncas ancestrales, parientes y vecinos de toda la vida, bandos y contrabandos sosteniendo una economía errática, movediza e informal que diferencia la zona del resto de cada uno de los países (s.f.).

En estas circunstancias, el portuñol como lenguaje de los colonos brasileños, está determinado por las condiciones históricas, económicas y sociales que lo interpela y por las relaciones entre los distintos sectores sociales en pugna por la hegemonía lingüística en la zona. Es resultado de préstamos, complicidades e interdependencias funcionales al poder (Alabarces y Añón, 2008), es resultante de procesos de interacción característicos de las zonas fronterizas como afirma Camblong, pero a la vez, resultado de procesos de exclusión permanente.

\section{El Brique}

Brique es una palabra brasilera que refiere al intercambio de objetos sin la intervención del dinero, lo que se conoce en otros lugares como trueque o cambalache. "Los briques son transacciones informales que se llevan a cabo entre conocidos, amigos, parientes, y pueden involucrar tierra, animales, automóviles y enseres domésticos" (Schiavoni, 2008b, p. 171), definición a la que, por mi trabajo de campo en la zona, agrego alimentos. La autora afirma que si bien se quiebra el "tabú del cálculo" entre los actores que producen el intercambio, la conveniencia final del trato deriva de la coyuntura y la oportunidad. Que una de las partes tenga urgencia en adquirir un determinado bien, interviene en la formación del valor.

La ausencia del dinero en la transacción no significa el desconocimiento del valor monetario, ya que se usa el dinero como referencia. La función que le corresponde al dinero en este tipo de transacciones es abstracta, deviene en medida de referencia aunque no sea utilizado como medio de pago. El dinero ha sido sustraído de los intercambios - como en su momento la sociedad europea lo hizo con el oro-; sin embargo las formas de dinero (en este caso los bienes) tienen valor en tanto que representan al dinero, es por ello que se le sigue teniendo como medida de referencia. El brique es un medio para la obtención de otro bien por lo que cumple una función semejante a la del dinero, que al realizarse en un círculo de conocidos y en un ámbito de mutuo conocimiento, reduce los costos de transacción. La relación entre vendedor y comprador es personal y hasta podría definirse como una práctica que se lleva a cabo preferencialmente entre conocidos y parientes, como afirma Schiavoni (2008b), donde se pone en juego la astucia de la negociación para la fijación del monto a intercambiar (que es variable), o mejor dicho, de los bienes que permitirán el trueque. En este tipo de transacciones la cercanía de los vínculos es una variable más para la conformación del precio. 
Esta forma de circulación de bienes, donde la característica esencial es la ausencia del dinero, coexiste con la forma mercantil de compra-venta de productos, es decir, se articulan dos modos de transacción uno tradicional y otro moderno. El brique no debe ser entendido en términos evolutivos, es decir, "no deriva necesariamente en mercado" (Schiavoni, 2008b, p. 179), sino que ambas formas de compra-venta conviven temporalmente. Esta convivencia de modos de circulación de las mercancías que parecerían contradictorios entre sí, permite paliar y compensar la escasa monetarización de las poblaciones que habitan la zona de frontera. "Para las fiestas antes teníamos que intercambiar mercadería en Brasil para comprar comida para navidad por ejemplo, no contábamos con efectivo casi” (Wolsky, 2015). Como evidencia este testimonio, el brique es una herramienta que surge justamente en un contexto donde el dinero circulante es prácticamente nulo. La pequeña producción familiar otorga la mayoría de los bienes necesarios para el autoconsumo del grupo doméstico haciendo escaso el uso del dinero y posibilitando el intercambio de excedentes entre las familias de productores.

Tener como referencia el dinero como medida de intercambio (a pesar que este no se encuentre en circulación) demuestra la utilización de prácticas dominantes transformadas y adecuadas a las clases populares. La cultura se hace de negociaciones y concesiones al interior de las relaciones de poder donde se hace uso de ciertas prácticas dominantes transformándolas y adaptándolas a las necesidades particulares del contexto.

Como afirman Alabarces, Moreira y Garriga (2012), la aparición de pautas culturales tiene estrecha relación con las condiciones económico-estructurales de la sociedad analizada. En el caso de las prácticas sociales y culturales estudiadas en este trabajo aparecen en un primer momento como manifestaciones de resistencia, aunque rápidamente se observa la estrecha relación que tienen con las prácticas hegemónicas. Frente a determinadas condiciones de existencia, estas prácticas pueden interpretarse como alternativas de supervivencia de las poblaciones rurales de frontera, lo que nos corrobora ese vínculo entre las pautas culturales y las condiciones estructurales que afirman estos autores. Estas prácticas no intentan modificar la posición de dominación sino nombrarla, hacerla visible y en cierto sentido "esquivarla": si no pueden comprar bienes a través del dinero, se crean prácticas alternativas que tienen el mismo fin (adquirir un bien) sin modificar la situación estructural. No se intenta disputar la hegemonía, pero sí conseguir el bien deseado (o al menos hacer más amena la dominación). Si analizamos estas prácticas en clave dominante, en tanto formas de intercambio alternativo que contribuye a la creación de un mercado informal (que no paga impuestos, por ejemplo) se podría reforzar el carácter resistente de estas formas de comercio.

Cabe entonces preguntarnos si el brique podría ser analizado como práctica de resistencia o como mera reproducción, de una forma de intercambio dominante que si bien no utiliza el dinero lo tiene como referencia para efectuar el cálculo. Parece ser una práctica ambigua y compleja — hasta podría definírsela como dialéctica- donde se manifiesta resistencia y a la vez reproducción.

\section{La compra-venta de mejoras: El mercado de tierras en el nordeste de Misiones}

La venta de mejoras es una de las formas principales de adquisición de parcelas de tierra en la frontera nordeste de Misiones, donde la ocupación del territorio se llevó a cabo de forma espontánea sobre tierras privadas. Como afirma Schiavoni (2008a) esta modalidad se pone en práctica sin la presencia de sistemas estandarizados de evaluación de los bienes — sin 
un mercado institucionalizado y sin la presencia del Estado-, siendo fruto de convenios particulares realizados entre el vendedor y el potencial comprador, especialmente entre primeros y segundos ocupantes. Responde a transacciones comerciales que están desconectadas de las demás, donde el precio depende de la relación entre vendedor y comprador, conformándose un mercado completamente personal, donde la tierra no es una mercancía entendida como las demás sujeta al libre juego de la oferta y la demanda, sino que circula dependiendo de la conveniencia mutua y la oportunidad. Lo que se comercializa es el trabajo incorporado en las parcelas "a la venta" aunque no se guíe por ningún sistema objetivo (Schiavoni, 2008a). El trabajo incorporado en las parcelas y la existencia de mensuras previas son dos mecanismos que generan un proceso de valorización de la tierra.

Ante la imposibilidad de acceder a la tierra en su país de origen, y frente a la inviabilidad de acceder al derecho (burgués) de propiedad privada en el país de destino, los migrantes ponen en funcionamiento ciertas prácticas que les permiten acceder a un mercado de tierras particular. La carencia de títulos de propiedad sobre la tierra, impide la venta de parcelas a través de un mercado institucionalizado, por lo que ponen a la venta el trabajo efectuado sobre los predios ocupados dado que el único bien ("legítimo") que poseen es la venta de su fuerza de trabajo. Muchas veces la venta de mejoras requiere un contrato sostenido en el tiempo donde por el trabajo efectuado en una determinada parcela de tierra (por ejemplo, la existencia de una producción de citronela en el predio destinado para la venta) el comprador debe otorgarle, por una cantidad de tiempo previamente acordada, cierto monto económico, aunque como queda reflejado en el siguiente testimonio, la transacción de compra-venta de mejoras no está exenta de problemas:

Una vez fui intermediario en un conflicto entre dos vecinos que habían entablado una compra-venta de chacras [de mejoras]. Uno había acordado por tantas semanas entregarle tanta cantidad de citronela. El tipo no la entregaba. Acordé que todos los viernes de 8 a 10hs tenía que dejar la citronela en la escuela y el otro vecino tenía que pasarla a buscar de 10 a $12 \mathrm{hs}$ por unas 20 semanas. Todo se arregló sin problemas, siguieron siendo buenos vecinos (Swinter, 2015).

Estos conflictos evidencian el carácter personal de las transacciones. En el mercado actual uno se ve imposibilitado de acceder al responsable del producto (pues no hay un único responsable sino muchos, tantos como lo determina la división del trabajo). Con suerte podrá recurrir al responsable del intercambio o algún organismo de defensa al consumidor. Se podrá visualizar cómo la compra-venta de mejoras muchas veces se entremezcla con el brique, mientras que otras veces con el pago mediado por el dinero.

La situación es similar al caso anteriormente analizado. Estas prácticas culturales de frontera implican la modificación y negociación con ciertas experiencias oficiales para hacerlas viables y adecuarlas a las posibilidades de los actores sociales analizados. Evidencian a la vez resistencia y reproducción de ciertas prácticas burguesas, pero explicitan la reacomodación de las mismas a las necesidades de los colonos.

\section{Las ocupaciones de tierras privadas}

En el nordeste de la provincia de Misiones entre la década de 1960 y 1990 se da un proceso de ocupación de tierras privadas bajo la modalidad de poblamiento espontáneo. Llegan al país un sinnúmero de inmigrantes brasileños imposibilitados de acceder a la tierra en Brasil por el proceso de modernización agrícola que atraviesa dicho país, 
dejando a grandes masas de población rural marginalizadas de este proceso. Los efectos socioeconómicos en las poblaciones de origen campesino son dramáticos: concentración de la propiedad de la tierra, regresión en la distribución de la renta, migraciones y éxodo rural, explotación de la fuerza de trabajo, profundización del proceso de expropiación de los campesinos, entre otros (Alentejano y Pereira, 2014). Esta situación se corresponde con un fuerte desequilibrio poblacional en la zona de frontera: se evidencia simultáneamente una saturación demográfica en las colonias del sur de Brasil, mientras que la frontera nordeste de la provincia de Misiones, hacia 1960, se encontraba deshabitada, con grandes extensiones de monte libre, como refleja el entrevistado: "allá había mucha gente, y acá era todo monte" (Unterhaun, 2015). El espacio rural se convierte en un factor de atracción para algunos inmigrantes limítrofes despojados de su tierra; la migración rural-rural se convierte en una estrategia de vida (Hughes, Sassone y Owen, 2007) y de reproducción social; entendida, en términos de Baudel (1996), como una maniobra de recampesinización ante ciertos procesos que tienden a desplazar al campesinado de las áreas rurales.

"Los brasileros vinieron por la tierra, allá no había y era cara, acá se metían y ocupaban. Hacían un rozadito y se metían. Así empezaron a poblar la zona. Después comenzaban a venir parientes, conocidos" (Herter, 2015). La llegada de migrantes se hace bajo la modalidad de ocupación silenciosa (Schiavoni, 2005) o en cadena como manifiesta un integrante del Movimiento Agrario Misionero (МАM) ${ }^{15}$ basada en relaciones de parentesco y amistad. Llegan primero algunos miembros de la familia, para después, una vez asentados traer a sus parientes y conocidos. Las familias ingresan y llevan adelante la ocupación mediante redes domésticas informales, sin un plan de acción organizado, donde se pone en juego un capital social que circula y produce saberes singulares (saber migrar).

La práctica de "tumba, roza y quema" determina la ocupación espontánea en esta zona de la provincia. La ocupación representa una vía alternativa para adquirir parcelas de tierra, ante la imposibilidad de este sector de comprar tierra en el mercado formal; es una estrategia que permite negociar el valor del terreno, jugar con los plazos y los medios de pago (Schiavoni, 2005).

Las ocupaciones de tierras privadas en Misiones pueden interpretarse como una estrategia tendiente a lograr adquisiciones inmediatas (en este caso, unidades domésticoproductivas donde llevar a cabo la pequeña producción familiar) evitando cualquier tipo de confrontación directa con las autoridades o los dueños de las tierras. Estas prácticas no tienden a lograr una mejora estructural de las condiciones de vida campesinas, sino acceder a mejoras parciales que permitan hacer más soportable la pobreza y la opresión. Crea intersticios en los espacios de poder con el objetivo de obtener demandas concretas, en este caso, la tierra.

La práctica de ocupación espontánea, se conforma a partir de las condiciones estructurales de un sector de la población rural excluida del acceso a la tierra. Los colonos no se oponen al derecho de propiedad de la tierra, pues después de ocuparla muchos de ellos tienen como objetivo conseguir primero el permiso de ocupación y luego el título de propiedad. No es una práctica social que se opone al derecho (burgués) de propiedad de la tierra sino que es una estrategia para acceder a él, al igual que la compra-venta de mejoras.

Como se describió en este artículo, las prácticas de intercambio (brique, compra-venta de mejoras, ocupaciones) no siempre se llevan a cabo de forma fragmentada. La mayoría de las veces se entremezclan entre sí:

${ }^{15}$ Creado en agosto de 1971, organización que compone las Ligas Agrarias del Nordeste. 
Iba y en una naciente de agua hacia un rozadito de media hectárea y le vendía a Blanca y Blanca traía a sus parientes y decía che ahí hay tierra buenísima. Ni compraba, venia y ocupaba. Y así en 2, 3 años se llenó de gente que no había lugar para nadie. Mis cuñados y suegros se hicieron así. Había algunos que tenían alguna mejora y compraban, otros cambiaban por animales, hacían cambalache, briquete... Acá suele haber mucho cambalache, brique. Acá estaba Mariana, Blanca, Elio que eran filosos para vender chacra, que hacían una chacrita y vendían. Entonces yo, yo no conocía la empresa el Soberbio S. A. por decirte pero Mariana si sabía y me dijo che allá hay para ocupar y te vendo la chacra (Queirós, 2015).

Esta situación evidencia la accesibilidad al mercado informal de la tierra en el nordeste de Misiones, entendiendo que existen prácticas para todas las situaciones socioeconómicas posibles. Aquellos que no cuentan ni con bienes ni con cierto monto en dinero llevan a cabo las ocupaciones; el que dispone de bienes y encuentra un vendedor que esté interesado en intercambiar una parcela de tierra por los bienes disponibles, lleva adelante el brique; aquellos que cuentan con un monto en dinero pero no es suficiente para comprar la totalidad del lote, combinan el brique y la compra de mejoras, y aquellos que disponen de dinero realizan únicamente esta última transacción. Por último, para quienes disponen de medios económicos, el mercado formal de tierras permite el acceso al título de propiedad correspondiente, forma de intercambio poco habitual entre los migrantes brasileños de la zona analizada.

\section{Reflexiones finales}

La cultura popular no puede escapar a una lectura relacional para con la cultura dominante, en tanto refiere a una concepción del mundo y de la vida en contraposición a las concepciones oficiales. Como hemos analizado en este trabajo, las prácticas culturales de los migrantes brasileños de la zona de frontera demuestran préstamos, concesiones, amalgamas, conflictos, subordinación y a la vez creatividad con esa cultura hegemónica, a la que si bien no pueden acceder, hacen uso a través de reapropiaciones y reacomodamientos.

Ninguna de las prácticas analizadas pueden interpretarse como "puramente resistentes", pero todas ellas permiten visibilizar la dominación en la que se encuentran los sectores sociales estudiados. En el caso del brique y la compra-venta de mejoras, estas modalidades de intercambio permiten afrontar las condiciones estructurales de existencia en la frontera, lo que las vuelve en cierta forma prácticas de resistencia a pesar de utilizar modalidades de intercambio provenientes de los sectores dominantes. Las experiencias burguesas son adecuadas y transformadas a las posibilidades de los colonos. Lo mismo ocurre con las ocupaciones. Esta práctica no tiene como objetivo modificar la estructura agraria de la localidad ni tiene como objetivo principal expropiar a los dueños de la tierra, sino que el objetivo es acceder a una parcela de tierra con el fin de reproducir el estilo de vida campesino. Una vez ocupadas, lo productores anhelan acceder al permiso de ocupación y al título de propiedad. El brique, la compra-venta de mejoras y las ocupaciones, se asemejan a las formas cotidianas de resistencia definidas por Scott (2014) dado que si bien no tienden a lograr una mejora estructural, permiten acceder a mejoras parciales que permitan afrontar las condiciones de existencia en la frontera. En palabras de Stern (1990) serían formas de adaptación en resistencia resultado 
de una continua experimentación y acumulación de experiencia de sus relaciones con el Estado y los sectores no campesinos (latifundistas).

El caso del portuñol es mucho más complejo y ambiguo y hasta nos genera un profundo conflicto ideológico. Si bien su utilización implica la acentuación de condiciones de dominación —dado que limita a la población rural a trabajar al interior de su comunidad por desconocimiento del idioma "oficial", precisamente en la agricultura familiar, "en la chacra" o en todo caso en trabajos poco calificados y mal pagos del mercado- el portuñol representa intersticios de prácticas genuinas, autónomas que permiten el mantenimiento de sus costumbres y de la cultura colona. A pesar de ello, es imposible negar que la presencia de esta lengua en tanto única conocida y manejada por los colonos, sea consecuencia de una exclusión permanente.

Esta imposibilidad de definir las prácticas culturales de la frontera como puramente resistentes o autónomas demuestra el carácter relacional que posee la cultura popular para con la cultura dominante, lo que imposibilita el análisis aislado del fenómeno. La tensión que tratamos de develar entre resistencia o reproducción es característica de los estudios culturales. Llevar adelante un análisis relacional que haga hincapié en las relaciones de poder y dominación, no significa negar la capacidad que poseen los sectores analizados de rehuir a esa situación de dominación. La reapropiación del intercambio $a$ su imagen y semejanza podríamos definirlo en cierto sentido como una práctica liberadora, que manifiesta la capacidad creativa de estos sectores y la capacidad de generar un sistema simbólico coherente. Ninguna práctica cultural es autónoma, sería ilusorio pensarlo así; todas poseen - en mayor o menor medida - contacto con la cultura occidental y cristiana; el poder que posee esta última es tan inmenso, son tantos los recursos a su disposición y tan desventajosa la posición de la cultura subalterna, que es imposible que las experiencias de las clases populares no se encuentren mediadas por la relación con esa cultura dominante, lo que vuelve imprescindible poner en diálogo ambas perspectivas de análisis. La condición de dominación de la cultura popular es incuestionada y la capacidad de estos sectores para evadirla también. Pueden generarse concesiones, momentos de autonomía y creatividad pero nunca pierden su condición de subalternidad y de dominación. Como dice Camblong (s.f.), "habitar la frontera supone estar inmersos en un movimiento constante de mezclas, amalgamas, corrimientos, sustituciones, retorcimientos y cambios que derrapan entre idiomas, costumbres e imaginarios sin solución de continuidad", las mismas mezclas, amalgamas e interdependencias que caracterizan a la cultura popular. El análisis de una cultura de frontera no puede más que explicitar esas entremezclas, interacciones y tránsitos entre lenguajes, experiencias y prácticas cotidianas.

\section{Referencias}

Alabarces, P. y Añón, V. (2008). ¿Popular(es) o subalterno(s)? De la retórica a la pregunta por el poder. En P. Alabarces y M. Rodríguez (Comps.), Resistencias y mediaciones. Estudios sobre cultura popular (pp.281-303). Buenos Aires, Argentina: Paidós.

Alabarces, P., Añón, V. y Conde, M. (2008). Un destino sudamericano. La invención de los estudios sobre cultura popular en la Argentina. En P. Alabarces y M. Rodríguez (Comps.), Resistencias y mediaciones. Estudios sobre cultura popular (pp. 261-280). Buenos Aires, Argentina: Paidós.

Alabarces, P., Moreira, M. V. y Garriga, J. (2012). La cultura como campo de batalla. Futbol y violencia en la Argentina. Versión Nueva Época, (29). 
Alentejano, P. y Pereira, J. M. M. (2014). El agro brasileño: de la modernización conservadora a la hegemonía del agronegocio. En G. Almeyra, L. Concheiro, J. M. Méndez y C. W. Porto-Gonvalves (Coords.), Capitalismo: tierra y poder en América Latina (19822012) (pp. 63-130). Buenos Aires, Argentina: Ediciones Continente.

Aliano, N. (2010). Culturas populares. Orientaciones y perspectivas a partir del análisis de un campo de estudios. Sociohistórica, (27), 185-209.

Bartolomé, L. (1975). Colonos, plantadores y agroindustrias. Desarrollo Económico, 15(58), 239-264.

Bartolomé, L. (1982). Base social e ideología en las movilizaciones agraristas en Misiones entre 1971 y 1975 . Emergencia de un populismo agrario. Desarrollo Económico, 22(85), 25-56.

Baudel, M. (Octubre, 1996). Raízes históricas do campesinato brasileiro. XX Encontro anual da Anpocs GT 17. Processos Sociais Agrários, Caxambu, Minas Gerais.

Bourdieu, P. (2000). Los usos del pueblo. En P. Bourdieu (Ed.), Cosas dichas, (pp. 152158). Barcelona, España: Gedisa.

Camblong, A. (2005). Mapa semiótico para la alfabetización intercultural en Misiones. Posadas, Argentina: Facultad de Humanidades y Ciencias Sociales, Universidad Nacional de Misiones.

Camblong, A. (s.f.). Instalaciones en los umbrales mestizos criollos. Manuscrito inédito. Seminario de Políticas Lingüísticas, Universidad de Tres de Febrero, Sáenz Peña, Argentina.

Camblong, A. (Septiembre, 2014). Semiótica de fronteras: dimensiones y pasiones territoriales. En Foro Internacional Fronteras Culturales. Facultad de Arte, Diseño y Ciencias de la Cultura, Universidad Nacional del Nordeste, Corrientes, Argentina. Recuperado de http://www.artes.unne.edu.ar/documentos/Extension/Fronteras\% 20Culturales/4-Conferencia-de-Cierre/Ana\%20CAMBLONG-Semi\%C3\%B3ticas\%20de\%20Fronteras-pdf

Cicourel, A. (1982). La medida y las matemáticas. En A. Cicourel (Ed.), El método y la medida en Sociología (pp. 33-67). Madrid, España: Editora Nacional.

Cohen, N. (2009). Una interpretación de la desigualdad desde la diversidad étnica. En N. Cohen (Comp.), Representaciones de la diversidad: escuela, juventud y trabajo (pp. 1129). Buenos Aires, Argentina: Ediciones Cooperativas.

Gramsci, A. (2004). Antología. Buenos Aires, Argentina: Siglo XXI.

Hughes, J., Sassone, S. y Owen, O. (Octubre-noviembre, 2007). Migración y dinámicas rurales en el Valle Inferior del Río Chubut. IX Jornadas Argentinas de Estudios de Población, Huerta Grande, Córdoba, Argentina.

Instituto Nacional de Estadísticas y Censos [Indec]. (2012). Censo Nacional de Población, Hogares y Vivienda 2010: Censo del Bicentenario: Resultados Definitivos Serie B $N^{\circ} 2$. Buenos Aires, Argentina: Autor.

Pottilli, J., Silverstein, Y. y Tavernelli, R. (2009). De la jerarquización de naciones a la clasificación de sujetos: representaciones que perpetúan un orden exclusor. En N. Cohen (Comp.), Representaciones de la diversidad: escuela, juventud y trabajo (pp. 3064). Buenos Aires, Argentina: Ediciones Cooperativas.

Reboratti, C. (1979). Migraciones y frontera agraria: Argentina y Brasil en la cuenca del Alto Paraná-Uruguay. Desarrollo Económico, 19(74), 189-209.

Rodríguez, M. G. (2011). Cultura popular: mi pie izquierdo. Revista Oficios Terrestres, 1(26). 
Romano, E. (1973). Apuntes sobre cultura popular y peronismo. En N. Brisky, La cultura popular del peronismo (pp. 9-58). Buenos Aires, Argentina: Cimarrón.

Schiavoni, G. (2008a). Madereros y agricultores. La construcción de un mercado de tierras en el nordeste de Misiones. En G. Shiavoni, Campesinos y agricultores familiares. La cuestión agraria en Misiones a fines del siglo XX (pp. 149-169). Buenos Aires, Argentina: Ciccus.

Schiavoni, G. (2008b). Notas sobre el brique o negocio amistoso. En G. Shiavoni, Campesinos y agricultores familiares. La cuestión agraria en Misiones a fines del siglo XX (pp. 171185). Buenos Aires, Argentina: Ciccus.

Schiavoni, G. (1994). Colonos y ocupantes. Parentesco, reciprocidad y diferenciación social en la frontera agraria de Misiones. Posadas, Argentina: Contemporánea.

Schiavoni, G. (1998). Porto-Capiovara: los ocupantes agrícolas de la frontera agrícola- brasileña. Estudios Migratorios Latinoamericanos, 13-14(40-41), 449-469.

Schiavoni, G. (2005). La construcción de los 'sin tierra' en Misiones, Argentina. Revista Theomai. Estudios sobre Sociedad, Naturaleza y Desarrollo, (12). Recuperado de http:/ / www.redalyc.org/articulo.oa?id=12401201

Scott, J. (2014). Explotación normal, resistencia normal. Relaciones Internacionales, (26), 85-104.

Stern, S. (1990). Nuevas aproximaciones al estudio de la conciencia y las rebeliones campesinas: las implicancias de las experiencias andinas. En S. Stern (Comp.), Resistencia, rebelión y conciencia campesina en los Andes. Siglos XVIII al XX (pp. 25-41). Lima, Perú: Instituto de Estudios Peruanos.

Winikor, M. (2013). De prejuicios y disciplinamientos. La mirada docente y el rol de la escuela pública frente a los inmigrantes limítrofes que llegan a la Argentina. Integra Educativa. Revista de Investigación Educativa, vI(1), 44-55.

\section{Material legislativo}

Ley de Migraciones No. 25.781 (2004). Argentina.

\section{Entrevistas}

Herter, H. (10 de agosto de 2015). Entrevistas a colonos rurales. Entrevistador M. Winikor Wagner. El Soberbio, Misiones.

Queirós, E. (5 de noviembre de 2015). Entrevistas a dirigentes del mam. Entrevistador M. Winikor Wagner. El Soberbio, Misiones.

Swinter, F. (1 de junio de 2015). Entrevistas a nacidos y criados en El Soberbio. Entrevistador M. Winikor Wagner. El Soberbio, Misiones.

Unterhaun, O. (12 de mayo de 2015). Entrevistas a nacidos y criados en El Soberbio. Entrevistador M. Winikor Wagner. El Soberbio, Misiones.

Wolski, S. (5 de mayo de 2015). Entrevistas a colonos rurales. Entrevistador M. Winikor Wagner. El Soberbio, Misiones. 
Mariana Winikor Wagner

Argentina. Doctoranda en Antropología Social en la Universidad Nacional de Misiones (UNam). Licenciada en Sociología (2012) y Profesora de Enseñanza Secundaria, Media, Normal y Especial de Sociología de la Universidad de Buenos Aires (2014). Desde 2005 participa en el Grupo de Estudios sobre Migración, Población y Desarrollo perteneciente al Instituto de Investigaciones Gino Germani, Universidad de Buenos Aires (UBA) y en el proyecto "Alternativas de desarrollo rural y formas de organización social" de la UNam desde 2015. Investiga sobre temas relacionados a la sociología rural y las migraciones. 